\title{
Inhibiting Adatom Diffusion through Surface Alloying
}

\author{
Zhengzheng Chen, ${ }^{1}$ Nicholas Kioussis, ${ }^{1}$ King-Ning Tu, ${ }^{2}$ Nasr Ghoniem, ${ }^{3}$ and Jenn-Ming Yang ${ }^{2}$ \\ ${ }^{1}$ Department of Physics, California State University, Northridge, California 91330-8268, USA \\ ${ }^{2}$ Department of Materials Science, University of California, Los Angeles, California 90095-1600, USA \\ ${ }^{3}$ Department of Mechanical and Aerospace Engineering, UCLA, Los Angeles, California 90095-1597, USA
}

(Received 12 March 2010; revised manuscript received 1 June 2010; published 1 July 2010)

\begin{abstract}
$A b$ initio and kinetic Monte Carlo calculations elucidate the electronic nature of surface Sn alloying on the stability and mobility of a $\mathrm{Cu}$ adatom on the $\mathrm{Cu}-\mathrm{Sn}$ (111) alloy surface. $\mathrm{Sn}$ atoms segregate on the surface and introduce forbidden areas around them within which adatom adsorption is strictly prohibited. In addition they reduce dramatically both the binding and the mobility of $\mathrm{Cu}$ adatoms in neighboring adsorption sites outside the forbidden areas, in contrast to experimental suggestions. Thus, Sn atoms act as blocking sites inhibiting the $\mathrm{Cu}$ adatom diffusion. The underlying mechanisms are the structural deformation associated with the oversized $\mathrm{Sn}$ atoms and the enhancement of the adatom-surface interaction in the vicinity of Sn atoms.
\end{abstract}

DOI: 10.1103/PhysRevLett.105.015703

Surface electromigration (EM), involving the diffusion of atoms under the influence of an electric field along the direction of electron flow, is of a major concern for the reliability of integrated circuits which carry large current densities and may exhibit significant mass transport. This induces the formation and migration of voids at the cathode end of the interconnect, and hillocks at the anode end, leading eventually to failure of the conducting interconnects in microelectronics technology [1].

The microelectronics industry has recently turned to copper as the interconnect conductor, due to its superior performance and reliability, particularly in the context of its lower electrical resistivity and its intrinsically higher EM resistance [2]. These properties, combined with the recently discovered ultrahigh strength of nanotwin copper wires [3], offers a unique opportunity in producing freestanding copper interconnects (with air as the lowest $k$ dielectric) in ultralarge-scale integrated circuits. The EM induced mass transport paths in $\mathrm{Cu}$ interconnects at temperatures of $\leq 100^{\circ} \mathrm{C}$, and occurs preferentially on the (111) free surfaces or interfaces [4].

Surface alloying with various metallic elements [5-9] has been employed as an effective way to improve the EM performance of $\mathrm{Cu}$, with the heavy $p$-block elements, $\mathrm{Sn}$, $\mathrm{Pb}$, etc., being the best candidates. A $20 \mathrm{~nm}$ thick $\mathrm{Cu}_{3} \mathrm{Sn}$ intermetallic compound overlayer on the $\mathrm{Cu}$ surface was found to effectively block dominant surface diffusion paths, thus resulting in an EM lifetime improvement of close to 1 order of magnitude [9]. Recent progress in growth techniques of high-quality metallic surface alloys and in real-time microscopy techniques allowing the observation of the time dependence of surface structure in unprecedented detail offers a way to manipulate surface kinetics. However, in contrast to the well-studied surface self-diffusion (homoepitaxial) or the diffusion of atoms different from the substrate (heteroepitaxial), the atomistic mechanisms of kinetics in surface alloys, which can be
PACS numbers: 64.60.De, 05.60. $-\mathrm{k}$, 71.15.Mb, 73.20. $-\mathrm{r}$

much different from and more complicated than those on the clean surface, have not been explored adequately. Understanding the origin in the electronic structure of surface-atom transport in alloy surfaces may provide guiding rules for the design of novel interconnect materials with superior EM resistance properties [10]. However, identifying the kinetic processes in surface alloys is computationally extremely demanding, because of the complex diffusion energy landscape, inherent in multispecies atoms, involving numerous inequivalent migration paths [8]. Even though extensive experimental studies have been carried out on the effect of $\mathrm{Sn}$ alloying on the $\mathrm{Cu}$ surface [2,9], the underlying mechanisms that control the kinetics remain unresolved.

In this Letter, we employ $a b$ initio and kinetic Monte Carlo calculations to understand the electronic nature of surface Sn alloying on the stability and mobility of a $\mathrm{Cu}$ adatom on the $\mathrm{Cu}-\mathrm{Sn}$ (111) alloy surface, in the dilute $\mathrm{Sn}$ and ordered $\mathrm{Cu}_{3} \mathrm{Sn}$ limits. We demonstrate that substitutional $\mathrm{Sn}$ atoms segregate on the surface and introduce forbidden areas (FAs) around them within which adatom adsorption is strictly prohibited. Sn surface atoms also reduce dramatically both the binding and the mobility of $\mathrm{Cu}$ adatoms in their neighboring adsorption sites outside the FAs. The combination of these two effects renders Sn atoms blocking sites which inhibit the $\mathrm{Cu}$ adatom diffusion. The underlying mechanism is the interplay of (i) a structural effect associated with the oversized Sn atoms leading to severe atomic distortions, and (ii) an electronic effect associated with the enhancement of both the adatomsurface interaction and the local density of states (LDOS) at the Fermi energy $\left(E_{F}\right)$ in the vicinity of Sn.

The $a b$ initio calculations were carried out employing the VASP code $[11,12]$ with the generalized gradient approximation [13] for the exchange and correlation functional and the projected augmented wave approach [14] to represent the electron-ion interaction. The Brillouin-zone 
integration was performed with a $3 \times 3 \times 1 \quad k$-point Monkhorst-Pack mesh [15]. The $\mathrm{Cu}(111)$ surface was modeled employing the slab supercell approach consisting of five $5 \times 5$ and $7 \times 7$ atomic (111) layers and a $10.5 \AA$ thick vacuum region separating the periodic slabs. Optimized atomic geometries are achieved when forces on all the unconstrained atoms on the top three layers are smaller in magnitude than $0.02 \mathrm{eV} / \AA$. [16] The nudged elastic band method $[17,18]$ was used to compute the diffusion pathways and migration barriers of a $\mathrm{Cu}$ adatom on the $\mathrm{Cu}-\mathrm{Sn}$ (111) alloy surface. We have used three replicas between the initial and final geometries to produce a smooth minimum energy path.

The formation energy, $E_{f}$, of a substitutional $\mathrm{Sn}$ atom on the surface, subsurface, and third layer below the surface is $-0.81 \mathrm{eV}, 4.26 \mathrm{eV}$, and $4.4 \mathrm{eV}$, respectively, with the negative sign denoting stable formation. Thus, the $\mathrm{Sn}$ atom can be incorporated solely into the surface layer. In order to understand the nature of the interaction between two surface Sn atoms, we list in Table I the change in the total energy between two $n$th nearest-neighbor (NN) tins and that of the first $\mathrm{NN}$ as a function of their separation. We find that the first NN Sn-Sn pair is a highly unfavorable configuration, in agreement with experiment [19]. The trend of both $E_{f}$ and $\mathrm{Sn}-\mathrm{Sn}$ interaction can be attributed to the oversized $\mathrm{Sn}$ atoms which are $\sim 20 \%$ larger than $\mathrm{Cu}$ atoms. Thus, $\mathrm{Sn}$ atoms prefer to segregate to and protrude from the surface by $0.6 \AA$ to accommodate the size mismatch, creating a tensile strain in the surrounding region and a $\mathrm{Sn}-\mathrm{Sn}$ repulsion so as to decrease the elastic energy. The Sn-Sn interaction does not change appreciably beyond the 2nd-NN separation, indicating that for a fixed Sncoverage there are several stable configurations of $\mathrm{Sn}$ atoms on the alloy surface.

Figure 1 shows the energy landscape for adsorption of a $\mathrm{Cu}$ adatom on the $\mathrm{Cu}-\mathrm{Sn}$ (111) alloy surface which has two 4th-NN Sn atoms. The higher energy values in the vicinity of the $\mathrm{Sn}$ sites correspond to weaker $\mathrm{Cu}$ adsorption. The absence of local energy minima at the threefold hollow sites which are $\mathrm{NN}$ to $\mathrm{Sn}$ demonstrates that these sites become unstable for adsorption due to the protrusion of the Sns from the surface. Consequently, each $\mathrm{Sn}$ introduces FAs of radius $r_{f}=(\sqrt{2} / 2) a_{0}$ ( $a_{0}$ is the $\mathrm{Cu}$ lattice constant) centered on it, within which a $\mathrm{Cu}$ adatom is strictly prohibited to adsorb. Thus, Sn atoms act as adsorption blocking sites which impede the adatom diffusion. Similarly, the origin of the weaker adsorption sites in the vicinity of $\mathrm{Sn}$ lies on the larger size of the embedded Sn atoms which in turn repel their 1 st-NN $\mathrm{Cu}$ atoms and hence reduce the

TABLE I. Change in total energy between two $n$th NN Sn atoms and that of first $\mathrm{NN}$ as a function of their separation.

\begin{tabular}{lcccccc}
\hline \hline$n$th NN & 1 & 2 & 3 & 4 & 5 & 6 \\
\hline$\Delta E(\mathrm{meV})$ & 0 & -368 & -394 & -407 & -395 & -407 \\
\hline \hline
\end{tabular}

available volume for adsorption. Interestingly, while surface $\mathrm{Sn}$ atoms bind strongly with in-plane $\mathrm{NN} \mathrm{Cu}$ atoms leading to $E_{f}<0$, they do not bind with $\mathrm{NN} \mathrm{Cu}$ adatoms, contrary to experimental suggestions [9]. These results, consistent with those of Anderson et al. for the $\mathrm{Cu}-\mathrm{Pb}$ alloy surface [8], suggest that the size mismatch between the heavy $p$ block and $\mathrm{Cu}$ plays an important role in tailoring surface diffusivity.

We have investigated two adatom diffusion paths on the $\mathrm{Cu}$ (111) surface alloyed with two 4th-NN Sn surface atoms, shown in Fig. 2(a). The first (I) migration path is in the vicinity of only one Sn atom, while the second (II) one is in between the two Sn atoms, respectively. The large yellow (gray) circles denote the $\mathrm{Cu}(\mathrm{Sn})$ surface atoms, while the midsize (green) and small (red) circles denote hcp and fcc adsorption sites, labeled by Greek and Latin letters, respectively. The two dark shaded areas centered on each Sn atom denote the FAs. The landscape of the minimum energy path along the I and II pathways is shown in Fig. 2(b), where the initial adatom position is denoted by an arrow. The values of the diffusion barrier, $E_{m}$, bet ween various inequivalent fcc and hcp sites, not shown in Fig. 2(b), are listed in Table II. $E_{f}^{i}\left(E_{f}^{\sigma}\right)$ is 2.97 (2.96) eV, and $E_{m}^{d \leftrightarrow \delta}$ is $48.3 \mathrm{meV}$, both in agreement with the values for the clean $\mathrm{Cu}$ (111) surface. The $\mathrm{Sn}$ atoms have a substantial effect on both the potential energy and the migration energy of the adsorption sites which are within the light shaded region in Fig. 2(a). For both pathways, there is an increase (ranging from 60 to $130 \mathrm{meV}$ ) in the potential energy for $\mathrm{Cu}$ adsorption on the 2nd- and 3rd-NN sites $(\chi, c, \beta, e, \varepsilon, f, \lambda)$ with respect to the Sn atoms. In addition, the migration barrier between these fcc and hcp sites (outside the FAs) increases to $84-99 \mathrm{meV}$, clearly demonstrating that the $\mathrm{Cu}$ adatom mobility close to $\mathrm{Sn}$ is substantially reduced. The asymmetric shallow energy minima associated with the 3rd-NN hcp site suggests that at room temperature the $\mathrm{Cu}$ adatom can easily diffuse backward away from the Sn atom. The enhancement of the blocking propensity associated with the Sn pair is due

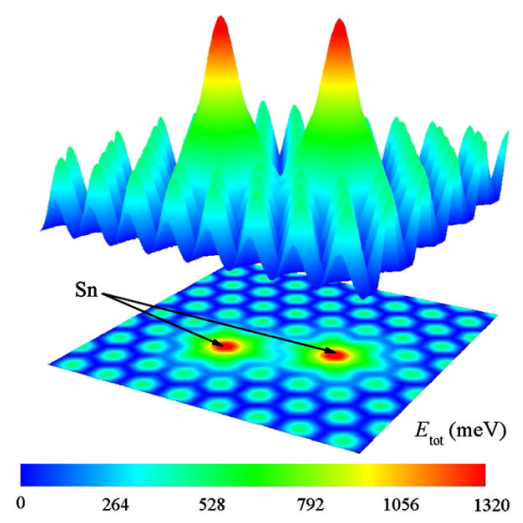

FIG. 1 (color online). Landscape of change in the total energy of a $\mathrm{Cu}$ adatom on the $\mathrm{Cu}$ (111) surface alloyed with two 4th-NN $\mathrm{Sn}$ atoms. The brighter (darker) regions denote weak (strong) adsorption sites. 


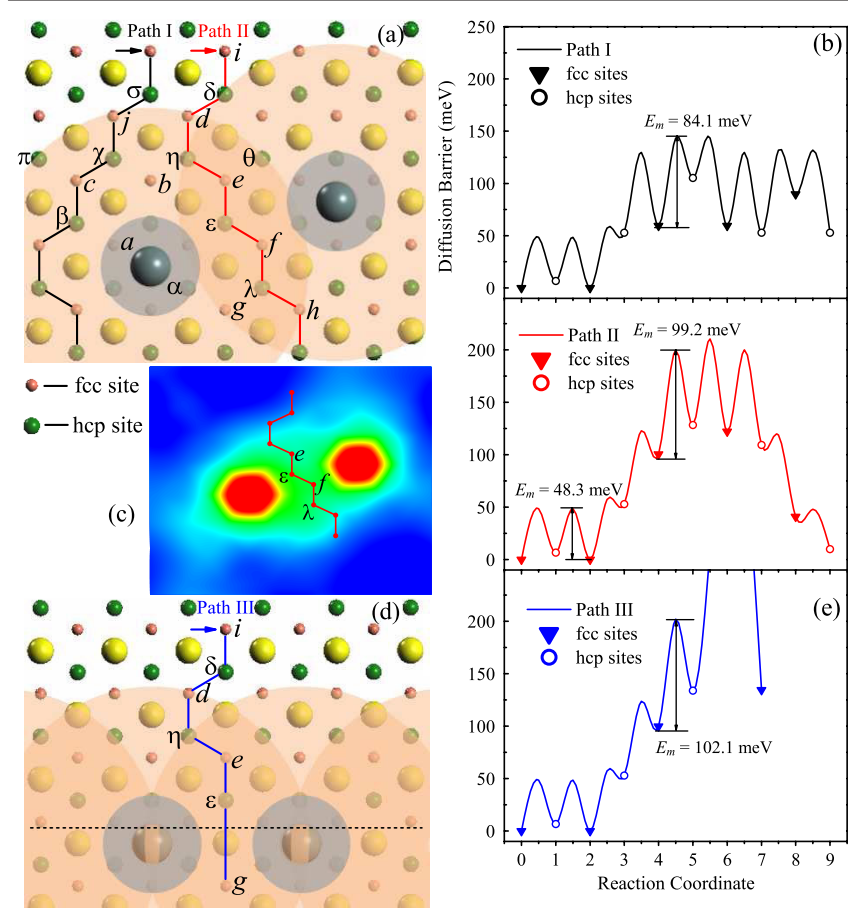

FIG. 2 (color online). (a) Migration paths I and II for a $\mathrm{Cu}$ adatom on the $\mathrm{Cu}-\mathrm{Sn}$ (111) alloy surface with two 4th-NN Sn surface atoms. Yellow (gray) circles denote the $\mathrm{Cu}(\mathrm{Sn})$ atoms, while green (red) circles labeled by Greek (Latin) letters denote hcp (fcc) sites. (b) Migration energy landscape for paths I and II versus reaction coordinates. (c) LDOS $\left(E_{F}\right)$ contour plot, where bright (dark) color indicates high (low) LDOS values. (d) and (e) Migration path and energy landscape for the $\mathrm{Cu}_{3} \mathrm{Sn} / \mathrm{Cu}$ interface denoted by dotted line.

to the concerted formation of low-mobility areas between Sn atoms.

The migration path (III) and corresponding energy landscape for the $\mathrm{Cu}_{3} \mathrm{Sn} / \mathrm{Cu}$ mixed surface is shown in Figs. 2(d) and 2(e), respectively. Because the $\langle 110\rangle \mathrm{Cu}-\mathrm{Sn}-$ $\mathrm{Cu}$ atomic rows in the ordered $\mathrm{Cu}_{3} \mathrm{Sn}$ surface consist of

TABLE II. Values of migration barrier, $E_{m}$, of various inequivalent paths of the $\mathrm{Cu}$ adatom on the $\mathrm{Cu}-\mathrm{Sn}$ surface alloy with two 4th-NN Sn atoms. The labels are shown in Fig. 2(a), and the superscripts "atop" and "ex" represent migration paths over the atop site or exchanges with a surface atom in the dilute and ordered alloy cases.

\begin{tabular}{|c|c|c|}
\hline Migration path & $E_{m}^{\mathrm{fcc} \rightarrow \mathrm{hcp}}(\mathrm{meV})$ & $E_{m}^{\mathrm{hcp} \rightarrow \mathrm{fcc}}(\mathrm{meV})$ \\
\hline fcc $\leftrightarrow$ hcp (pure $\mathrm{Cu})$ & 48.3 & 41.7 \\
\hline$a \leftrightarrow \beta$ & $\infty$ & $\infty$ \\
\hline$b \leftrightarrow \chi$ & 42.1 & 78.9 \\
\hline$c \leftrightarrow \pi$ & 7.0 & 36.5 \\
\hline$e \leftrightarrow \theta$ & 29.7 & 37.4 \\
\hline$g \leftrightarrow \lambda$ & 51.4 & 27.4 \\
\hline$b \stackrel{\text { atop }}{\leftrightarrow} \sigma$ & 427.7 & 421.1 \\
\hline$b \stackrel{\mathrm{ex}}{\leftrightarrow} \sigma$ & 1289.1 & 1282.5 \\
\hline$g \stackrel{\text { atop }}{\leftrightarrow} \varepsilon\left(\mathrm{Cu}_{3} \mathrm{Sn} / \mathrm{Cu}\right)$ & 443.1 & 442.3 \\
\hline
\end{tabular}

2nd-NN Sn atoms, both the connectivity of the FAs centered on $\mathrm{Sn}$ and the low-mobility areas between $\mathrm{Sn}$ atoms increase, leading to the blocking of the 1st-NN fcc $\leftrightarrow$ hcp $(\epsilon \rightarrow f, f \rightarrow \lambda$, etc.) migration paths available in the dilute case, thus reducing the diffusion dramatically.

Two important factors are responsible for the lowmobility areas around the Sn atoms (outside the FAs) in Fig. 2(b). First is that these are weak adsorption sites and second is the increase of $E_{m}$ between these sites. The former effect arises from the severe distortions of the atomic configurations due to the oversized $\mathrm{Sn}$ atoms. $A b$ initio calculations for the clean $\mathrm{Cu}$ surface constrained to have an identical atomic displacement field as the $\mathrm{Cu}-\mathrm{Sn}$ dilute alloy surface yields $E_{m}^{e \leftrightarrow \varepsilon}=45 \mathrm{eV}$, about half its value in Fig. 2(b), suggesting that the second effect is of electronic origin. Indeed we find that the strong $\mathrm{Sn} / p-\mathrm{Cu} / d$ surface hybridization (i) lowers and broadens the upper peak of the LDOS of the $\mathrm{Cu}$ surface atoms which are 1st-NN to $\mathrm{Sn}$ and (ii) increases the $\operatorname{LDOS}\left(E_{F}\right)$, of $s-p$ character, in the vicinity of Sn. Sn decreases (increases) the height (width) of the adatom's LDOS whose center shifts towards lower energy. Using the Newns-Anderson model [20] and the $a b$ initio determined LDOS, we find that the larger $E_{m}$ is due to the increase by a factor of 2 of the adatom- $d$-surface- $d$ interaction in the vicinity of Sn atoms. Moreover, the increase (decrease) of the LDOS $\left(E_{F}\right)$, also entering the Newns-Anderson model, from $i(f)$ to $f(h)$ along path II correlates well with the variation of $E_{m}$ in Fig. 2(c), suggesting that the confinement of the surface electrons may also play a role [21].

Using the $a b$ initio calculated $E_{m}$ of all inequivalent paths, we have carried out kinetic Monte Carlo simulations $[22,23]$ to study the temperature-dependent diffusion of a $\mathrm{Cu}$ adatom on (i) the dilute Sn-pair alloyed surface, and (ii) the $\mathrm{Cu}_{3} \mathrm{Sn} / \mathrm{Cu}$ surface consisting of an ordered $\mathrm{Cu}_{3} \mathrm{Sn}$ and a clean $\mathrm{Cu}$ phase. The probability distribution for a $\mathrm{Cu}$ adatom adsorption for these two cases is shown in Figs. 3(a)-3(c) and in Figs. 3(d)-3(f) at $300 \mathrm{~K}, 600 \mathrm{~K}$

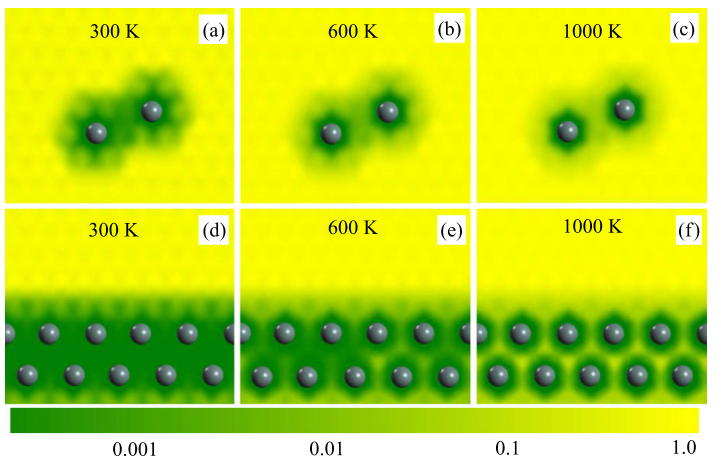

FIG. 3 (color online). Probability distribution (logarithmic scale) for a $\mathrm{Cu}$ adatom on the $\mathrm{Cu}$ (111) surface at $300 \mathrm{~K}$, $600 \mathrm{~K}$, and $1000 \mathrm{~K}$, respectively, for (i) the dilute Sn-pair alloyed system [(a)-(c)], and (ii) the surface consisting of an ordered intermetallic $\mathrm{Cu}_{3} \mathrm{Sn}$ and a clean $\mathrm{Cu}$ phase $\left(\mathrm{Cu}_{3} \mathrm{Sn} / \mathrm{Cu}\right)$ [(d)-(f)]. The gray circles denote $\mathrm{Sn}$ atoms. 
and $1000 \mathrm{~K}$, respectively. Employing ab initio calculations and the harmonic transition state theory [23], the diffusion attempt frequency $\nu_{0}=1.6 \mathrm{THz}$ for clean $\mathrm{Cu}$.

In the dilute Sn-pair alloy, the diffusion channel for the $\mathrm{Cu}$ adatom between the two $\mathrm{Sn}$ atoms is closed at $300 \mathrm{~K}$ due to the FAs and low-mobility areas between the Sn atoms in Fig. 2(a). Thus, the $\mathrm{Sn}$ pair hinders the $\mathrm{Cu}$ adatom diffusion redirecting it away from or around it. This diffusion path opens with increasing temperature. Nevertheless, even at $1000 \mathrm{~K}$, where the migration rate $\nu^{\mathrm{fcc} \leftrightarrow \mathrm{hcp}}$ is reduced by a factor of 2 compared to that in clean $\mathrm{Cu}$, the blocking effect of $\mathrm{Sn}$ remains noticeable.

The blocking effect of $\mathrm{Sn}$ on adatom diffusion becomes much highly pronounced in the $\mathrm{Cu}_{3} \mathrm{Sn} / \mathrm{Cu}$ surface, shown in Figs. 3(d)-3(f). Because of the exclusion of the 1st-NN paths, the $\mathrm{Cu}$ adatoms are forced to diffuse solely through atop site and/or exchange migration paths $[b \leftrightarrow \sigma$ or $g \leftrightarrow$ $\varepsilon$ in Figs. 2(a) and 2(d) and Table II], with an energy barrier of about $15 \mathrm{meV}$ higher than in pure $\mathrm{Cu}$. Thus, Sn surface atoms increase not only the energies of both the fcc and hcp sites but also the energy at the saddle points due to the fact that the adatom is farther away from the surface. The migration rates of these two paths, of $\sim 10^{-7} \mathrm{THz}$ and $\sim 10^{-21} \mathrm{THz}$ at $300 \mathrm{~K}$, respectively, are extremely low compared with that of $0.25 \mathrm{THz}$ on the clean $\mathrm{Cu}$ (111) surface, thus inhibiting the adatom diffusion on the $\mathrm{Cu}_{3} \mathrm{Sn}$ phase. Even though more diffusion channels become available at $600 \mathrm{~K}$, the average probability distribution for adatom adsorption remains low. At $1000 \mathrm{~K}$ the thermal energy of the adatom is large enough to overcome the migration barrier of the 2nd-NN fcc $\leftrightarrow$ hcp path and the migration rates $\nu^{\mathrm{fcc} \leftrightarrow \mathrm{hcp}}$ of the two paths increase to $\sim 10^{-2} \mathrm{THz}$ and $\sim 10^{-6} \mathrm{THz}$, respectively, which, however, are much smaller than in pure $\mathrm{Cu}$. Consequently, at $1000 \mathrm{~K}$ the $\mathrm{Cu}$ adatom diffuses in the $\mathrm{Cu}_{3} \mathrm{Sn}$ phase, but with an extremely low mobility. The average lifetime of the $\mathrm{Cu}$ adatom on the available adsorption sites in $\mathrm{Cu}_{3} \mathrm{Sn}$ is $\sim 200$ times longer than that in pure $\mathrm{Cu}$, indicating that the ordered $\mathrm{Cu}_{3} \mathrm{Sn}$ structure is an excellent electromigrationinhibiting candidate.

In summary, we have shown that substitutional surface Sn atoms introduce FAs within which adatom adsorption is strictly prohibited. Even though surface $\mathrm{Sn}$ atoms bind strongly with $\mathrm{NN}$ surface $\mathrm{Cu}$ atoms, they do not bind with the $\mathrm{NN} \mathrm{Cu}$ adatoms, in contrast to experimental suggestions. Sn surface atoms reduce dramatically both the binding and the mobility of $\mathrm{Cu}$ adatoms in neighboring adsorption sites outside the FAs. Thus, Sn atoms act as blocking sites impeding the $\mathrm{Cu}$ adatom diffusion. The underlying origin is the structural deformation associated with the large size of Sn atoms and the enhancement of the adatom-surface interaction in the vicinity of Sn. The monolayer or few layers thick $\mathrm{Cu}_{3} \mathrm{Sn}$ surface enhances further the blocking propensity, rendering it an excellent overlayer candidate in improving the surface EM resistance of $\mathrm{Cu}$ interconnects. This work opens up the possibility of controlling adatom diffusion through selective alloying with various elements in the $p$ block.

We acknowledge valuable discussions with A.R. Akbarzadeh and the support by NSF-NIRT Grant No. CMS-0506841 and NSF-PREM Grant No. DMR00116566.

[1] A. V. Vairagar et al., Appl. Phys. Lett. 85, 2502 (2004).

[2] K. N. Tu, J. Appl. Phys. 94, 5451 (2003).

[3] L. Lu, Y. Shen, X. Chen, L. Qian, and K. Lu, Science 304, 422 (2004).

[4] K. C. Chen, W. W. Wu, C. N. Liao, L. J. Chen, and K. N. Tu, Science 321, 1066 (2008).

[5] P. J. Ding, W. A. Lanford, S. Hymes, and S. P. Murarka, J. Appl. Phys. 75, 3627 (1994).

[6] C. K. Hu and B. Luther, Mater. Chem. Phys. 41, 1 (1995).

[7] C. Cabral, J. M. E. Harper, K. Holloway, D. A. Smith, and R. G. Schad, J. Vac. Sci. Technol. A 10, 1706 (1992).

[8] M.L. Anderson, N. Bartelt, P. Feibelman, B. Swartzentruber, and G. Kellogg, Surf. Sci. 600, 1901 (2006).

[9] M. Y. Yan, J. O. Suh, F. Ren, and K. N. Tu, Appl. Phys. Lett. 87, 211103 (2005).

[10] E. T. Ogawa, K. D. Lee, V. A. Blaschke, and P. S. Ho, IEEE Trans. Reliab. 51, 403 (2002).

[11] G. Kresse and J. Hafner, Phys. Rev. B 47, 558(R) (1993).

[12] G. Kresse and J. Furthmüller, Phys. Rev. B 54, 11169 (1996).

[13] J. P. Perdew, K. Burke, and M. Ernzerhof, Phys. Rev. Lett. 80, 891 (1998).

[14] P. E. Blöchl, Phys. Rev. B 50, 17953 (1994).

[15] H. J. Monkhorst and J. D. Pack, Phys. Rev. B 13, 5188 (1976).

[16] Results for a $8 \times 8$ supercell show that the outermost shell atomic displacements are $\leq 0.008 \AA$ and the largest energy difference in Table I is $2 \mathrm{meV}$.

[17] G. Henkelman, B.P. Uberuaga, and H. J. Jónsson, J. Chem. Phys. 113, 9901 (2000).

[18] D. Sheppard, R. Terrell, and G. Henkelman, J. Chem. Phys. 128, 134106 (2008).

[19] A. K. Schmid, N. C. Bartelt, and R. Q. Hwang, Science 290, 1561 (2000).

[20] A. Groß, in Theoretical Surface Science: A Microscopic Perspective (Springer, Berlin, 2009), 2nd ed., p. 112.

[21] N. N. Negulyaev, V. S. Stepanyuk, L. Niebergall, P. Bruno, W. Hergert, J. Repp, K.-H. Rieder, and G. Meyer, Phys. Rev. Lett. 101, 226601 (2008).

[22] M. Ziegler, J. Kröger, R. Berndt, A. Filinov, and M. Bonitz, Phys. Rev. B 78, 245427 (2008).

[23] A. F. Voter, in Radiation Effects in Solids, edited by K. E. Sickafus, E. A. Kotomin, and B.P. Uberuaga (Springer, NATO Publishing Unit, Dordrecht, The Netherlands, 2006), p. 1. 